\title{
Concurrent Nanopore Next-Generation Sequencing of Hepatitis B and Delta Virus Genomes Directly From Patient Plasma
}

Philippe Colson (1), Pharm, D., Ph.D. ${ }^{1,2}$, Céline Boschi $\mathbb{B}^{1,2}$, Jessica Grace Bengone-Abogourin $\mathbb{0}^{1,2}$, Ludivine Brechard $\mathbb{0}^{2}$, Anne Motte (10, M.D. ${ }^{2}$, and Isabelle Allemand (iD, M.D. ${ }^{3}$

${ }^{1}$ Aix-Marseille Université, Institut de Recherche pour le Développement (IRD), Assistance Publique - Hôpitaux de Marseille (AP-HM), MEPHI, Marseille, France; ${ }^{2} \mathrm{H} \mathrm{HU}$ Méditerranée Infection, Marseille, France; ${ }^{3}$ Assistance Publique - Hôpitaux de Marseille (AP-HM), Marseille, France

\section{Dear Editor,}

The advent of next-generation sequencing (NGS) techniques has considerably improved the detection and characterization of microbial and viral genetic sequences. Although initially used to study environmental, animal, and human samples, NGS has been more recently introduced in clinical microbiology and virology laboratories [1-5]. Its technical requirements, processing time, and cost are now compatible with diagnostic use. NGS allows identification of infectious agents based on complete genomes and not only genes or gene fragments. Particularly the Oxford Nanopore Technology (ONT; Oxford, UK) permits extremely promising sequencing speed $[3,6]$. ONT allows singlemolecule sequencing of DNA or RNA fragments. Native DNA or RNA molecules are pulled through nanoscale pores one molecule at a time. While the nucleic acid passes through the pore, sensors detect changes in the ionic current that are specific to each passing nucleotide, which allows to determine the nucleic acid sequence.

While NGS is generally used following specific target enrichment, it can be applied directly to nucleic acids extracted from clinical samples. We recently used the latter approach in our clinical microbiology/virology laboratory (IHU Méditerranée Infection, Marseille) to detect and characterize genomes of hepa- titis B virus (HBV) by Illumina NGS (Illumina, Inc., San Diego, CA, USA) for the first time and of SARS-CoV-2 by both Illumina and ONT NGS [4, 7]. Compared with Sanger population sequencing, NGS provides information on mutations, deletions, and insertions for all viral genes simultaneously as well as for intergenic regions, and therefore allows a more comprehensive and deeper assessment of viral genomes. Such comprehensive data can contribute to the elucidation of the genome features associated with various clinical characteristics, which, in the case of HBV infection, include acute liver failure, drug resistance, immune escape, or viral reactivation. Here, we describe NGS of a fulllength HBV genome and a partial hepatitis delta virus (HDV) genome directly from a single patient plasma sample using ONT GridION. The ethics committee of IHU Méditerranée Infection, Marseille, France, approved this study (No. 2019-001).

In June 2020, we obtained a plasma sample from a 27-yearold male patient to monitor his HBV and HDV infections diagnosed in October 2018 during a systematic screening. He was not aware of viral hepatitis in his family. It is unknown whether he was co-infected with HBV and HDV or experienced HDV superinfection on chronic HBV infection. In November 2018, liver ultrasound testing showed normal results, transient elastography showed no liver fibrosis, and the patient was clinically asymp-
Received: November 7, 2020

Revision received: December 4, 2020

Accepted: May 10, 2021

Corresponding author: Philippe Colson, Pharm.D., Ph.D.

IHU Méditerranée Infection, 19-21 boulevard Jean Moulin, 13005 Marseille, France

Tel: +33-413-732-401; Fax: +33-413-732-402

E-mail: philippe.colson@ap-hm.fr

\section{(c) (1) $(9$}

\section{(C) Korean Society for Laboratory Medicine}

This is an Open Access article distributed under the terms of the Creative Commons Attribution Non-Commercial License (https://creativecommons.org/licenses/by-nc/4.0) which permits unrestricted non-commercial use, distribution, and reproduction in any medium, provided the original work is properly cited. 
tomatic.

In June 2020, ALT and AST levels were $87 \mathrm{IU} / \mathrm{L}$ and $115 \mathrm{IU} / \mathrm{L}$, respectively (normal upper values, $50 \mathrm{IU} / \mathrm{L}$ ). Serological testing showed hepatitis B surface (HBs) antigen (Ag)-positivity, total anti-HB core antibody ( $\mathrm{Ab}$ )-positivity, anti-HBs Ab-negativity, hepatitis $\mathrm{B}$ e ( $\mathrm{HBe}) \mathrm{Ag}$-positivity, and anti-HBe Ab-negativity (Architect assays, Abbott Diagnostics, Mannheim, Germany). The serum HBV DNA load was $8.0 \log _{10} \mathrm{IU} / \mathrm{mL}$ (NeuMoDx HBV Quant Assay, Qiagen, Courtaboeuf, France). Anti-HDV IgG and IgM testing (Euroimmun, Lubeck, Germany) were both positive, and the HDV RNA load determined by an in-house PCR assay as previously described [8] was $9.6 \log _{10}$ copies/mL. We used ONT
GridION as previously described [7] to obtain HBV and HDV genome sequences without pre-PCR amplification. Briefly, DNA and RNA were extracted from $200 \mu \mathrm{L}$ of plasma using the EZ1 Virus Mini Kit v2.0 on a BioRobot EZ1 workstation (Qiagen), and RNA was reverse-transcribed using SuperScript IV (Thermo Fisher Scientific, Waltham, MA, USA) before cDNA second-strand synthesis with Klenow Fragment DNA polymerase (New England Biolabs, Beverly, MA, USA). The DNA was then purified using Agencourt AMPure XP beads (Beckman Coulter, Villepinte, France) and sequenced using the ONT Ligation Sequencing Kit on a GridION X5 instrument (Oxford Nanopore Technologies Ltd.). Genome consensus sequences were generated with the CLC
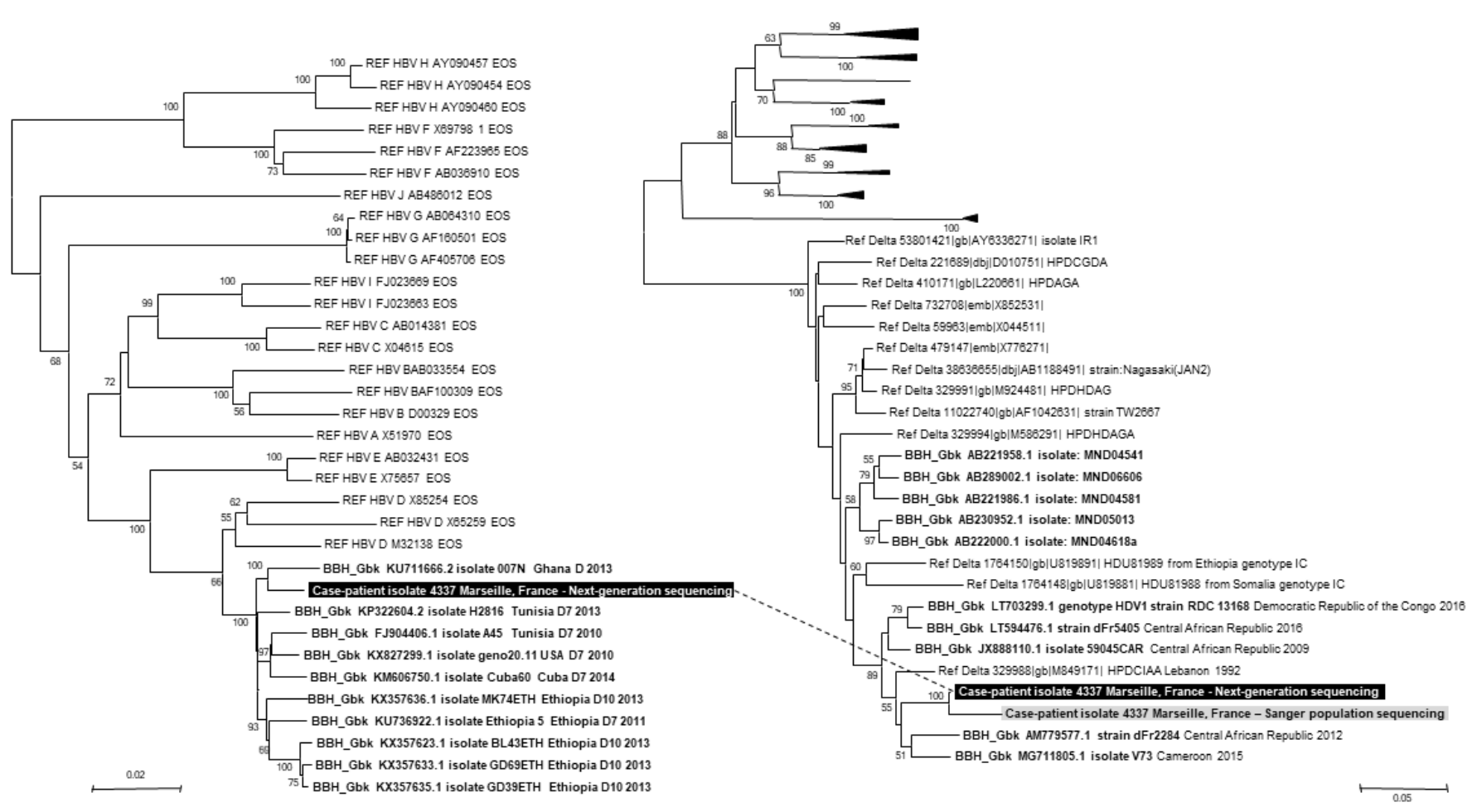

Fig. 1. Phylogenetic trees based on the HBV genome and HDV antigen-encoding gene. Phylogenetic reconstruction based on HBV genome sequences involved 3,252 positions in the final dataset, and phylogenetic reconstruction based on the HDV antigen-encoding gene involved 343 positions corresponding to nucleotides 824-1,154 of the HDV genome (GenBank accession no. AM779577.1). Viral sequences obtained from the patient's plasma using ONT (available from https://www.mediterranee-infection.com/acces-ressources/donnees-pourarticles/) are indicated by a white bold font on a black background, and those obtained by Sanger population sequencing are indicated by a black bold font on a grey background. HBV and HDV sequences obtained from the patient using ONT are connected by a dashed line. The 10 sequences with the highest BLAST scores recovered from the NCBI GenBank nucleotide sequence database (http://www.ncbi.nIm. nih.gov/nucleotide/), labeled with BBH GbK and indicated by a black bold font, were incorporated in the phylogeny reconstructions, in addition to reference sequences (indicated by Ref for Reference) for HBV or HDV genotypes. Nucleotide alignments were performed using the MUSCLE software (http://www.ebi.ac.uk/Tools/msa/muscle/). Evolutionary history was inferred using the MEGA6 software (http://www. megasoftware.net/) using the neighbor-joining method and the Kimura 2-parameter method. The percentage of replicate trees in which the associated taxa clustered together in the bootstrap test (1,000 replicates) is shown next to the branches. The tree is drawn to scale, with branch lengths in the same units as those of the evolutionary distances used to infer the phylogenetic tree; the scale bars indicate the number of nucleotide substitutions per site. Bootstrap values $>50 \%$ are indicated on the tree.

Abbreviations: HBV, hepatitis B virus; ONT, Oxford Nanopore Technology; NCBI, National Center for Biotechnology Information; BBH GbK, best BLAST hit GenBank. 
Genomics Workbench v.7 (https://digitalinsights.qiagen.com/ products-overview/discovery-insights-portfolio/analysis-and-visualization/qiagen-clc-genomics-workbench/) by mapping to HBV and HDV reference genomes (GenBank accession numbers M32138 and M84917, respectively), with $0.85 \%$ similarity and $50 \%$ coverage as thresholds. All data were generated as part of the routine study at Assistance Publique-Hôpitaux de Marseille (APHM), and this study results from routine standard clinical management. Access to the patients' biological and registry data issued from the hospital information system was approved by the data protection committee of APHM and was recorded in the European General Data Protection Regulation registry under number RGPD/APHM 2019-73.

In total, 41 (average length, 918 nucleotides) and three (average length, 490 nucleotides) reads were identified as belonging to HBV and HDV, respectively, generating full-length HBV and partial HDV genomes with a size of 3,179 and 326 nucleotides, respectively (available from https://www.mediterranee-infection. com/acces-ressources/donnees-pour-articles/hbv-hdv-ngs/). Phylogenetic analyses performed using the MEGA6 software (http://www.megasoftware.net/) identified HBV of genotype D and HDV of genotype 1 (Fig. 1). The mean diversity per nucleotide position of the HBV genome was $5.4 \% \pm 9.0 \%$, and the mean coverage was $8.9 \pm 2.9$. Neither anti-HBV drug resistance mutations nor HBsAg escape variants were detected. A 570-nucleotide-long sequence corresponding to the HDV Ag-encoding gene generated by Sanger population sequencing as previously described [8] clustered with that obtained using ONT GridION in the phylogenetic tree (Fig. 1).

We report for the first time, to the best of our knowledge, the concurrent detection and determination of HBV and HDV sequences, including full HBV genome recovery, on the same day and directly from nucleic acids extracted from plasma. Illumina or ONT NGS has been previously used to obtain HBV or HDV sequences, but generally only after target enrichment, which is more time-consuming [2, 9]. NGS directly from DNA or RNA extract without PCR amplification, as was done in the present case, is simpler and faster and allows simultaneous detection of several non-targeted infectious agents. Furthermore, NGS using ONT GridION allows viral genome detection and characterization within a timeframe that is appropriate for clinical diagnostic purposes. Thus, although still in its infancy, with necessary improvements in sensitivity and precision, ONT should optimize the diagnosis of infections in the near future.

\section{AUTHOR CONTRIBUTIONS}

Conceived and designed the study: Colson P. Contributed materials/analysis tools: Colson $\mathrm{P}$, Boschi $\mathrm{C}$, Bengone-Abogourin JG, Brechard L, Allemand I, Motte A. Analyzed the data: Colson P, Boschi C, Bengone-Abogourin JG. Wrote the paper: Colson P, llemand $\mathrm{A}$.

\section{CONFLICTS OF INTEREST}

None declared.

\section{FUNDING}

This work was supported by the French Government under the "Investments for the Future" program managed by the National Agency for Research, Méditerranée-Infection 10-IAHU-03 and by Région Provence-Alpes-Côte d'Azur and European funding FEDER PRIMMI (Fonds Européen de Développement RégionalPlateformes de Recherche et d'Innovation Mutualisées Méditerranée Infection), FEDER PA 0000320 PRIMMI.

\section{ORCID}

Philippe Colson

https://orcid.org/0000-0001-6285-0308

Céline Boschi https://orcid.org/0000-0003-2504-7828

Jessica Grace Bengone-Abogourin

https://orcid.org/0000-0002-8657-4624

Ludivine Brechard

https://orcid.org/0000-0003-1529-3830

Anne Motte https://orcid.org/0000-0002-8622-531X

Isabelle Allemand https://orcid.org/0000-0002-3514-4552

\section{REFERENCES}

1. Mitchell SL and Simner PJ. Next-generation sequencing in clinical microbiology: are we there yet? Clin Lab Med 2019;39:405-18.

2. Astbury S, Costa Nunes Soares MM, Peprah E, King B, Jardim ACG, Shimizu JF, et al. Nanopore sequencing from extraction-free direct PCR of dried serum spots for portable hepatitis B virus drug-resistance typing. J Clin Virol 2020;129:104483.

3. Moon J, Jang Y, Kim N, Park WB, Park KI, Lee ST, et al. Diagnosis of Haemophilus influenzae pneumonia by Nanopore $16 \mathrm{~S}$ amplicon sequencing of sputum. Emerg Infect Dis 2018;24:1944-6.

4. Colson P, Borentain P, Ravaux I, Aherfi S. Hepatitis B virus genomics knocking at the door of routine diagnostic laboratories. J Infect Dis 2020; 221:1026-9.

5. Zhong Y, Xu F, Wu J, Schubert J, Li MM. Application of next generation sequencing in laboratory medicine. Ann Lab Med 2021;41:25-43.

6. Quick J, Grubaugh ND, Pullan ST, Claro IM, Smith AD, Gangavarapu K, 
et al. Multiplex PCR method for MinION and Illumina sequencing of Zika and other virus genomes directly from clinical samples. Nat Protoc 2017; 12:1261-76.

7. Colson P, Lagier JC, Baudoin JP, Bou Khalil J, La Scola B, Raoult D. UItrarapid diagnosis, microscope imaging, genome sequencing, and culture isolation of SARS-CoV-2. Eur J Clin Microbiol Infect Dis 2020;39: 1601-3.
8. Petit PR, Borentain P, Aherfi S, Gérolami R, Colson P. Hepatitis Delta recurrence post-liver transplantation in absence of detectable hepatitis B surface antigen and hepatitis B virus DNA in peripheral blood. Clin Res Hepatol Gastroenterol 2020;44:e41-4.

9. McNaughton AL, Roberts HE, Bonsall D, de Cesare M, Mokaya J, Lumley SF, et al. Illumina and Nanopore methods for whole genome sequencing of hepatitis B virus (HBV). Sci Rep 2019;9:7081. 